\title{
INFLUENCE OF TWINNING ON LOCAL ELECTRONIC PROPERTIES OF ZnSe*
}

\author{
M. Balcerzyk, M. Grinberg and J. Szatkowski \\ Institute of Physics, N. Copernicus University \\ Grudziądzka 5/7, 87-100 Toruń, Poland
}

We have obtained $\mathrm{ZnSe}$ single crystals by vertical Bridgman method. The surface was examined using scanning electron microscope (SEM). As usual crystals exhibited twins, which were observed on the (110) surface. On as-grown and $n$-type samples we observed long (of the order of millimetres) linear structures parallel to the (111) plane which correspond to the twin plane. Our observations and measurements enabled us to estimate the electronic potential as being of the order of a few volts and extending less than $1 \mu \mathrm{m}$ in the direction perpendicular to the twin planes.

PACS numbers: $61.70 . \mathrm{Ng}, 71.55 . \mathrm{Gs}, 72.80 . \mathrm{Ey}$

Twining is a common structural defect in the production of $\mathrm{ZnSe}$ crystals which extends on available (111) twin plane in the crystal [1]. Some authors predict the mechanism due to which twins may control the type and the value of material conductivity. In 1983 Fitzpatrick et al. [2] showed that macroscopic defects (including twins) may remove compensating centres in $\mathrm{ZnSe}$. Wojtowicz and $\mathrm{Ma}$ linski [3], who investigated the polarization spectra of $\mathrm{Ag}$ in $\mathrm{ZnSe}$, suggested that the observed polarization peaks result from the additional electric field related to the charge of the twin plane. The aim of this work is the direct observation of this field. We examined the (110) surface of $\mathrm{ZnSe}$ crystal (the surface perpendicular to the twin planes) using SEM and transmission electron microscope (TEM).

We obtained $\mathrm{ZnSe}$ single crystals using vertical Bridgman method. The density of twins along [111] direction is about $300 / \mathrm{mm}$. The crystals were broken along cleavage plane $\{110\}$. The resistivity of one set of the samples was reduced in molten zinc [4], the samples were kept in $1233 \mathrm{~K}$ for 48 hours. The resistivity of the samples received was $0.2 \Omega \mathrm{cm}$. A small gold dot with sharp edges on (110) surface of some samples was evaporated. The typical view of high resistivity sample is presented in Fig. 1. This is a view of mirror like surface of (110) plane. The lines are parallel to [112] direction which is parallel to (111) plane. They form sharp lines, alternately lighter and darker than the area between them. The areas between the lines are of the same black colour. The resolution of that

* This work was in part supported by N. Copernicus University grant no. 615F and by Young Researcher Fund of N. Copernicus University Torun, Poland. 


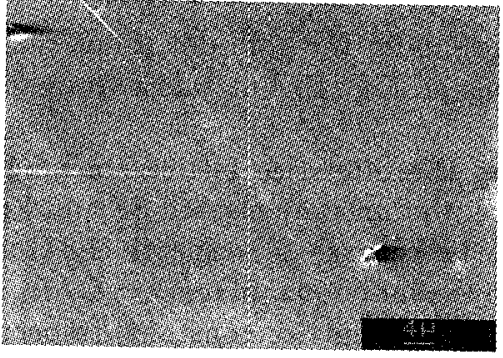

a

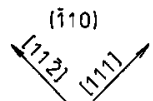

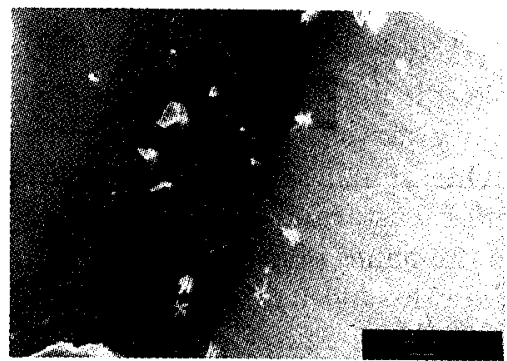

b

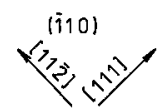

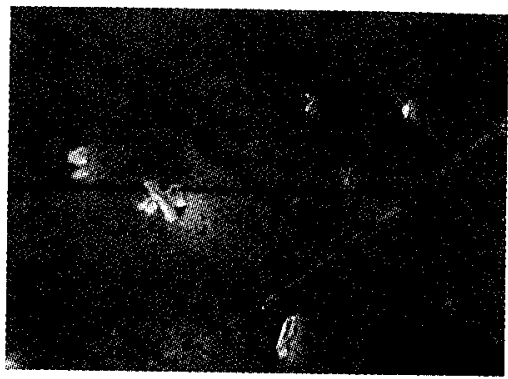

c

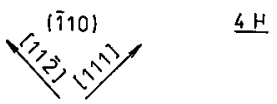

Fig. 1. The scanning electron microscope photograph of the (110) surface of the ZnSe crystal. The diagonal lines correspond to the crossing of (111) and (110) planes; (a) high resistivity crystal, (b) low resistivity crystal. The black area in the upper left corner is the fragment of the $0.1 \mu \mathrm{m}$ thick gold layer, (c) low resistivity crystal.

photograph is $0.5 \mu \mathrm{m}$. We made the replica of the surface in synthetic resin and observed it using TEM. The resolution of that method and of TEM was $0.1 \mu \mathrm{m}$. The observation yielded no vertical structure of the surface. So we attribute the structures in Fig. 1a to potential wells along [111] direction. This is reinforced by the same kind of observations on low resistivity samples (Fig. 1b). A small gold dot with sharp edges was evaporated on (110) surface of some samples. The area in the upper left corner corresponds to the gold dot. There is no structure to be seen. Then along [111] direction the structure appears with a small intermediate area. The gold wafer is $0.1 \mu \mathrm{m}$ thick and thus if the observed structure originates from vertical morphology it should be still observed with the gold coverage. The structure disappears and we attribute this to the flattening of the (111) surface potential by the presence of thin metallic layer on the surface. The same kind of structure on low resistivity materials looks somewhat different (Fig. 1c). Being of the same density and size along [111] direction the structures form alternate light 
and dark stripes. The structure also vanishes under the gold dot if one takes the photograph made under the same conditions as Fig. 1b but taken from the low resistivity samples.

One can interpret the structure using the interstice lattice stacking fault model of Austerman and Gechman [5]. Here, the uncompensated Se and $\mathrm{Zn}$ ions create the panes of negative $(N)$ and positive $(P)$ charge [6], respectively. One may calculate the density of the charge related to the individual plane $\rho=Q / a^{2}$, where $Q$ and $a$ is the charge of the $\mathrm{Zn}$ or Se ion and the lattice constant, respectively. Assuming further that each of charged planes is compensated by the bulk charge on the distance $d$ one obtains the parabolic potential given by the formula

$$
V= \begin{cases}{[\rho /(\varepsilon d)](|x|-d)^{2},} & |x|<d \\ 0, & |x| \geq d\end{cases}
$$

where $\varepsilon$ is dielectric constant. Knowing $Q$ and $d$ it is possible to estimate the maximum value of this potential. Assuming that the charges of the ions may be given by ionicity of the crystal, one may use for $Q$ the value calculated by Resta and Baldereschi [7] (for $\mathrm{ZnSe}$ the average value $Q= \pm 1.59$ [8]). Assuming $d=10 \mathrm{~nm}$ one obtains the maximum potential about ten volts. In fact the real potentials seem to be lower due to rearrangement of the valence electrons forming the bounds on the plain what yields the diminishing of the ionic charge by the factor of ten.

We would like to thank Dr W. Marciniak for making the replica of the surface and performing TEM observations.

\section{References}

[1] M. Balcerzyk, unpublished.

[2] B. Fitzpatrick, G.F. Neumark, R. Bhargava, Physica B 116, 487 (1983); G.F. Neumark, G.-J. Yi, B.J. Fitzpatrick, Bull. Am. Phys. Soc. 33, 694 (1988); G.F. Neumark, J. Appl. Phys. 65, 4859 (1989) and references therein.

[3] A.J. Wojtowicz, M.A. Maliniski, J. Cryst. Growth 72, 167 (1985).

[4] M. Aven, H.H. Woodbury, Appl. Phys. Lett. 1, 53 (1962).

[5] S.B. Austerman, W.G. Gechman, J. Mater. Sci. 1, 249 (1966).

[6] M. Shiojiri, C. Kaito, S. Sekimoto, N. Nakamura, Philos. Mag. A 46, 495 (1982).

[7] R. Resta, A. Baldereschi, Phys. Rev. B 24, 4839 (1981).

[8] M. Grinberg, Phys. Status Solidi B 145, 619 (1988). 